\title{
Subspace-Based Blind Channel Estimation for OFDM by Exploiting Virtual Carriers
}

\author{
Chengyang Li, Member, IEEE, and Sumit Roy, Senior Member, IEEE
}

\begin{abstract}
Reliable channel estimation is indispensable for orthogonal frequency-division multiplexing (OFDM) systems employing coherent detection and adaptive loading in order to achieve high data rate communications. Several options exist in practical OFDM systems-including training symbols, cyclic prefix, virtual carriers, pilot tones, and receiver diversity-to facilitate channel estimation. In this paper, a subspace blind channel estimation method based on exploiting the presence of virtual carriers is proposed for OFDM systems over a time-dispersive channel. The method can be applied to conventional OFDM systems with cyclic prefix as well as OFDM systems with no cyclic prefix. The reduction/elimination of cyclic prefix thereby provides the OFDM systems the potential to achieve higher channel utilization than most previously reported cyclic prefix based estimators. Sufficient channel identifiability condition is developed as well. Comparison with two other recently reported subspace methods is presented via computer simulations to support the effectiveness of the proposed method.
\end{abstract}

Index Terms-Blind channel estimation, cyclic prefix, orthogonal frequency-division multiplexing (OFDM), subspace approach, virtual carriers.

\section{INTRODUCTION}

$\mathbf{O}$ RTHOGONAL frequency-division multiplexing [1], [2] $(\mathrm{OFDM})$ is a promising candidate for high-data-rate wireless communications due to its many advantages-notably, its high spectral efficiency, robustness to frequency selective fading, as well as the feasibility of low-cost transceiver implementations. It is the basis of the European standard for digital audio/video broadcasting (e.g., DAB and DVB-T, target rates 1.7 and $20 \mathrm{Mb} / \mathrm{s}$, respectively) and is being developed internationally for use in high-speed wireless LANs (e.g., IEEE 802.11a [3], target rates 6-54 Mb/s) and wireless-local-loop applications (1-10 Mb/s).

Coherent detection and adaptive loading (see also [1]) in support of high speed data communications require reliable estimation of the channel. In practical OFDM systems operating over a dispersive channel, a cyclic prefix (CP) longer than the anticipated multipath channel spread is usually inserted in the transmitted sequence. It is well known that this converts the linear (time-domain) convolution between the channel and the input

Manuscript received March 24, 2001; revised December 12, 2001; accepted August 24, 2002. The editor coordinating the review of this paper and approving it for publication is C. Tellambura. This work was supported in part by the Air Force Office of Scientific Research under Grant F49620-1-0472 and the National Science Foundation under Grant CCR-0086032. Part of this work was presented at the Globecom 2001, San Antonio, TX, November 2001.

The authors are with the Department of Electrical Engineering, University of Washington, Seattle, WA 98195-2500 USA (e-mail: cyli@ee.washington.edu; roy@ee.washington.edu).

Digital Object Identifier 10.1109/TWC.2002.806383 into cyclic convolution or equivalently a (complex) multiplicative factor on each sub channel in the frequency domain. This naturally facilitates computationally simple frequency domain channel estimation by inserting a training sequence to estimate the factor on each sub channel; see [2], [4] for an overview and [7], [8] for recent results on these lines.

However, CP-based systems incur a price: significant loss of channel utilization that may be the overriding constraint for future high-speed services. That provides the motivation for methods such as those presented in this work. Usually, the length of $\mathrm{CP}$ is conservatively chosen according to the maximum anticipated multipath spread; for the IEEE 802.11a standard, this is $25 \%$ of an OFDM symbol duration. Additionally, due to the time-varying nature of the channel in some wireless applications (i.e., those that seek to provide mobility support), the training sequence needs to be transmitted periodically, causing further loss of channel throughput. Consequently, there exists increasing interest in OFDM systems with short or no CP. For example, the work [5] and [6] addressed this issue and proposed multiple-input/multiple-output (MIMO) equalization scheme for OFDM systems without $C P$.

\section{Literature Review}

The above concerns naturally leads to efforts centered around blind channel estimation methods that avoid the need for training and/or even the CP. The presence of CP has been utilized to devise methods for blind or semi-blind channel estimation in [9]-[14]. Among these, the statistically inspired blind estimators in [9] and [10] rest on the inherent CP-induced cyclostationarity at the transmitter explicitly or implicitly, while the estimators in [12] and [13] belong to the class of deterministic subspace approach. Specifically, Heath and Giannakis [9] proposed a blind method based on the cyclostationarity property of the time-varying correlation of the received data samples due to the CP insertion at the transmitter; however, this approach suffers from slow convergence of the estimator. Cai $e t$ al. [13] developed a noise subspace method [15] by utilizing the structure of the filtering matrix introduced by the $\mathrm{CP}$ insertion that achieves faster convergence for smaller data records.

Other than the CP, there exists another resource that has not been fully exploited for purposes of channel estimation-the presence of the virtual carrier (VC), ${ }^{1}$ as in the IEEE 802.11a standard that specifies 12 (out of a total of 64 subcarriers) VCs. While they are intended to aid in shaping of the transmit spectrum, the VCs can be exploited for the purposes of

\footnotetext{
${ }^{1}$ In practical fast Fourier transform (FFT)-based OFDM systems, a shaping filter is required for spectral masking, i.e., to limit the transmitted signal spectrum to the desired band. In order to ease filter implementation, some sub carriers in the roll-off region, namely the band edge of the filter are left unmodulated; these are referred to as virtual carriers [4].
} 


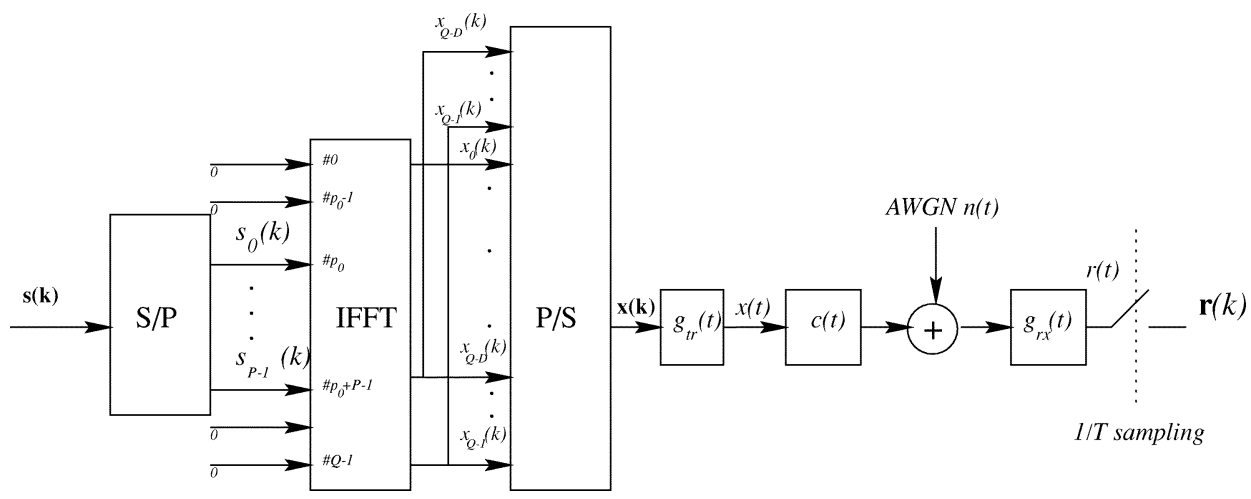

Fig. 1. Baseband OFDM system (with VC) model.

channel equalization or frequency offset estimation, as have already been shown in [16] and [17]. A method that exploits the presence of the VCs and the finite alphabet property of the input data was presented in [18] to develop a maximum likelihood (ML) joint blind estimator of the channel and the data symbols. In other related work, [19] reported a subspace channel estimator based on a multichannel model for non-CP OFDM systems with receiver oversampling/diversity which achieves performance comparable to [13], and, recently, [20] showed a novel blind channel estimator relying on the finite alphabet property of the information-bearing symbols.

\section{Specific Contribution}

In this paper, we propose a new subspace-based (blind) channel estimator for OFDM systems that exploits VCs and is applicable to OFDM systems with and without CP. For the former (conventional CP systems), the exploitation of VC brings additional performance gain to the already proposed blind estimators such as in [13]. Further, the proposed method can be employed for non-CP systems where CP-based estimators cannot be used, thereby potentially achieving higher channel utilization.

The rest of the paper is arranged as follows. A generalized baseband signal model for the OFDM system with both VC and CP is introduced in Section II. The subspace channel estimator is developed in Section III, where a sufficient condition on the channel identifiability is provided. Computer simulations are presented in Section IV to demonstrate the effectiveness of the proposed algorithm in comparison to other two subspace methods [9], [13]. Finally, Section V concludes the paper.

The notation used in this paper follows the usual convention-vectors are denoted by symbols in boldface and $(\cdot)^{*},(\cdot)^{T}$, $(\cdot)^{H}$ are complex conjugate, transpose, and conjugate transpose of $(\cdot)$, respectively. $\operatorname{rank}(\cdot)$ yields the rank of $(\cdot)$. range $(\cdot)$ and $\|\cdot\|$ give respectively the range and Frobenius norm of matrix argument. $\otimes$ stands for the Kronecker product. $A\left(i_{1}: i_{2}, j_{1}: j_{2}\right)$ denotes a submatrix obtained by extracting rows $i_{1}$ through $i_{2}$ and columns $j_{1}$ through $j_{2}$ from matrix $A$. If no specific range appears at the row or column position in notation $A\left(i_{1}: i_{2}, j_{1}\right.$ : $\left.j_{2}\right)$ [e.g., $A\left(:, j_{1}: j_{2}\right)$ or $A\left(i_{1}: i_{2},:\right)$ ], then all rows or columns are accounted for constituting the submatrix.

\section{SignAl Formulation}

Consider an OFDM system (see Fig. 1) with $Q$ subcarriers, of which only $P$ are modulated by the user's data symbols; i.e., the remaining $Q-P$ unmodulated carriers constitute VCs. Assume that the subcarriers numbered $p_{0}$ to $p_{0}+P-1$ are used for data, where $p_{0}$ is the index of the first data carrier. Also assume that the length of CP is $D$. Let the $k$ th block of the "frequencydomain" information symbols be

$$
\mathbf{s}(k)=\left[s_{0}(k), s_{1}(k), \ldots, s_{P-1}(k)\right]^{T} .
$$

Define a $Q \times Q$ IDFT matrix as

$$
\mathbf{W}_{Q}=\frac{1}{\sqrt{Q}}\left[\begin{array}{cccc}
1 & 1 & \cdots & 1 \\
1 & w_{Q}^{-1} & \cdots & w_{Q}^{-(Q-1)} \\
1 & w_{Q}^{-2} & \cdots & w_{Q}^{-2(Q-1)} \\
\vdots & & & \vdots \\
1 & w_{Q}^{-(Q-1)} & \cdots & w_{Q}^{-(Q-1)(Q-1)}
\end{array}\right]
$$

with $w_{Q}=e^{-j 2 \pi / Q}$. Multicarrier modulation, which is implemented by IFFT, yields the "time-domain" signal vector $\left[x_{0}(k), x_{1}(k), \ldots, x_{Q-1}(k)\right]^{T}=\mathbf{W} \mathbf{s}(k)$, where $\mathbf{W}$ is the $Q \times P$ partial IDFT matrix

$$
\mathbf{W}=\left[\begin{array}{llll}
1 & & & \\
& w_{Q}^{-p_{0}} & & \\
& & \ddots & \\
& & & w_{Q}^{-p_{0}(Q-1)}
\end{array}\right] \cdot \mathbf{W}_{Q}(:, 1: P) .
$$

CP insertion replicates the last $D$ elements of the IFFT output vector in the front and results a $J \times 1(J=Q+D)$ OFDM symbol vector

$$
\begin{aligned}
\mathbf{x}(k) & =\left[x_{Q-D}(k), \ldots, x_{Q-1}(k), x_{0}(k), x_{1}(k), \ldots, x_{Q-1}(k)\right]^{T} \\
& =\underbrace{\left[\begin{array}{c}
\mathbf{W}(Q-D+1: Q,:) \\
\mathbf{W}
\end{array}\right]}_{\overline{\mathbf{W}}} \mathbf{s}(k)=\overline{\mathbf{W}} \mathbf{s}(k)
\end{aligned}
$$


Each element or "chip" in the vector $\mathbf{x}(k)$ is then pulse shaped by $g_{t r}(t)$ to generate the continuous time signal sent on the channel

$$
x(t)=\sum_{k=-\infty}^{+\infty} \sum_{p=0}^{J-1} x_{p}(k) g_{t r}(t-(p+k J) T)
$$

where $T$ is the chip period. Thus, denoting $q=p+k J$, we identify $k=\lfloor q / J\rfloor(\lfloor x\rfloor$ is the largest integer contained in $x)$ and $p=q$ modulo $J$. Then the transmitted signal $x(t)$ can be rewritten as

$$
x(t)=\sum_{q=-\infty}^{+\infty} x_{q} g_{t r}(t-q T)
$$

where $x_{q}$ is an equivalent transmitted "chip" sequence corresponding to the $x_{p}(k)$ 's.

During the transmission, the signal $x(t)$ passes through a dispersive channel with impulse response $c(t)$, is contaminated by AWGN noise $n(t)$, and finally is input into a front-end receive filter $g_{r x}(t)$.

Defining the composite channel filter $h(t)=g_{t r}(t) * c(t) *$ $g_{r x}(t)$ and the filtered noise $v(t)=n(t) * g_{r x}(t)$ where $*$ denotes linear convolution, the received signal $r(t)$ is therefore

$$
r(t)=\sum_{q=-\infty}^{+\infty} x_{q} h(t-q T)+v(t) .
$$

Assume the composite channel $h(t)$ to have finite support $[0, L T]$ where $L<J$ (i.e., it is assumed that the channel delay spread does not exceed the OFDM symbol duration); this implies that any intersymbol interference (ISI) is only restricted to the past neighboring symbol as is generally true for OFDM. A synchronized rate $1 / T$ sampler after $r(t)$ yields $\left(t_{0}\right.$ denotes the sampling phase)

$$
r(i)=r\left(t_{0}+i T\right)=\sum_{l=0}^{L} x_{i-l} h\left(t_{0}+l T\right)+v(i)
$$

where $v(i)=v\left(t_{0}+i T\right)$.

Let $h(l)=h\left(t_{0}+l T\right)$ and the channel vector

$$
\mathbf{h}=[h(L), h(L-1), \ldots, h(0)]^{T} .
$$

Define an $M J-L \times M J$ Toeplitz matrix $\mathcal{H}$ constructed from h as

$$
\begin{aligned}
\mathcal{H} & =\text { Toeplitz }\left([\mathbf{h}^{T} \quad \underbrace{0 \cdots 0}_{(M J-L-1)}]\right) \\
& =\left[\begin{array}{cccccc}
h(L) & \cdots & h(0) & & & \\
& h(L) & \cdots & h(0) & & \\
& & \ddots & & \ddots & \\
& & & h(L) & \cdots & h(0)
\end{array}\right] .
\end{aligned}
$$

Consider an observation interval over $M$ OFDM symbols from $\left(t_{0}+((k-M+1) J+L) T\right)$ to $\left(t_{0}+((k+1) J-1) T\right)$. The resulting received signal vector $(M J-L \times 1)$ is

$$
\begin{aligned}
\mathbf{r}_{M}(k) & =[r((k-M+1) J+L), \ldots, r((k+1) J-1)]^{T} \\
& =\mathcal{H}\left[\begin{array}{c}
\mathbf{x}(k-M+1) \\
\vdots \\
\mathbf{x}(k-1) \\
\mathbf{x}(k)
\end{array}\right]+\underbrace{\left[\begin{array}{c}
v((k-M+1) J+L) \\
v((k+1) J-1)
\end{array}\right]}_{\mathbf{n}(k)} \\
& =\mathcal{H} \cdot \underbrace{\left(\mathbf{I}_{M} \otimes \overline{\mathbf{W}}\right)}_{\tilde{\mathbf{W}}} \cdot \underbrace{\left[\begin{array}{c}
\mathbf{s}(k-M+1) \\
\mathbf{s}(k-1)
\end{array}\right]+\mathbf{n}(k)}_{\mathbf{S}(k)} \\
& =\underbrace{\mathcal{H}}_{\mathcal{A}(k)} \mathbf{S}(k)+\mathbf{n}(k)
\end{aligned}
$$

where $\mathbf{I}_{M}$ is an $M \times M$ identity matrix.

Remarks: For the signal model (11) where the equivalent filtering matrix $\mathcal{A}$ has dimensions $M J-L \times M P$, a necessary condition for the subspace method is that $M(Q+D-P) \geq L$. Note that the presence of VCs implies $Q>P$ and the necessary condition can always be satisfied by choosing an appropriate $M$, which is true even for non-CP OFDM systems. This condition is assumed to hold throughout the paper.

\section{SubsPaCE-BASED ChANNEL ESTIMATION}

In this section, we develop a sufficient identifiability condition for the proposed subspace-based channel estimator.

\section{A. Sufficient Conditions for Identifiability}

From (11), $\mathcal{A}=\mathcal{H} \tilde{\mathbf{W}}(M J-L \times M P)$ where $\mathcal{H}$ is an $M J-$ $L \times M J$ Toeplitz matrix and $\tilde{\mathbf{W}}=\mathbf{I}_{M} \otimes \overline{\mathbf{W}}$ is an $M J \times M P$ matrix. We first demonstrate necessary and sufficient conditions for the filtering matrix $\mathcal{A}$ to have full column rank in Theorem 1 , as a preliminary step toward the (sufficient) identifiability condition in Theorem 2.

Theorem 1: For $Q+D-L \geq P, \mathcal{A}$ has full column rank (i.e., $\operatorname{rank}(\mathcal{A})=M P$ ) if and only if the channel frequency response has no nulls at any of the data subcarrier frequencies.

Proof: See Appendix B.

In the derivation hereafter, we assume that the above conditions are satisfied. Also, the user's transmitted information symbols $s_{i}(k)$ s are i.i.d. sequences with zero mean and known variance $\sigma_{s}^{2}$ ( $\sigma_{s}^{2}=1$ without loss of generality). In addition, Nyquist pulse shaping is employed so that each element of $\mathbf{n}(k)$ in (11) is additive white Gaussian noise (AWGN) with zero mean and variance $\sigma_{n}^{2}$. After collecting $N_{b}$ signal vectors, we have

$$
\begin{aligned}
\mathbf{Y} & =\left[\mathbf{r}_{M}(1), \ldots, \mathbf{r}_{M}\left(N_{b}\right)\right] \\
& =\mathcal{A} \underbrace{\left[\mathbf{S}(1), \ldots, \mathbf{S}\left(N_{b}\right)\right]}_{\mathbf{S}}+\mathbf{N}=\mathbf{X}+\mathbf{N} .
\end{aligned}
$$


Applying the singular value decomposition (SVD) on the unperturbed received signal matrix $\mathbf{X}$ yields

$$
\mathbf{X}=\mathcal{A} \mathbf{S}=\left[\begin{array}{ll}
\mathbf{U}_{s} & \mathbf{U}_{n}
\end{array}\right]\left[\begin{array}{ll}
\Sigma_{s} & \\
& \mathbf{0}
\end{array}\right]\left[\begin{array}{c}
\mathbf{V}_{s}^{H} \\
\mathbf{V}_{n}^{H}
\end{array}\right]
$$

where $\left[\mathbf{U}_{s} \mathbf{U}_{n}\right]$ is an $M J-L \times M J-L$ unitary matrix. The $M P$ columns of $\mathbf{U}_{s}$ span the signal subspace, while $M(Q+D-$ $P)-L$ column vectors of $\mathbf{U}_{n}$ span the subspace (often known as the noise subspace as in practice the SVD is applied on the noise perturbed signal matrix $\mathbf{Y}$ ) orthogonal to the signal subspace. $\boldsymbol{\Sigma}_{s}=\operatorname{diag}\left(\lambda_{1}, \lambda_{2}, \ldots, \lambda_{M P}\right)$ is a diagonal matrix consisting of $M P$ significant singular values corresponding to the signal subspace. The orthogonality property between the signal subspace and the noise subspace asserts that

$$
\left(\mathbf{U}_{n}(i)\right)^{H} \mathcal{A}=0 \quad i=1, \ldots, M(Q+D-P)-L
$$

where $\mathbf{U}_{n}(i)$ is the $i$ th column of $\mathbf{U}_{n}$.

The set of constraints (14) suggests a possible way to identify the channel vector. However, asserting the uniqueness of the resulting estimate of $\mathbf{h}$ (up to a complex scaling factor) requires some care since $\mathcal{A}$ is the product of an $M J-L \times M J$ matrix $\mathcal{H}$ and an $M J \times M P$ matrix $\tilde{\mathbf{W}}$. The following theorem provides a characterization of conditions under which $\mathbf{h}$ can be uniquely identified.

Theorem 2 (Sufficient Condition for Identifiability): Let $\mathbf{h}^{\prime}$ and $\mathbf{h}$ be distinct $L+1$-dimension vectors and $\mathcal{A}^{\prime}$ be a matrix constructed using $\mathbf{h}^{\prime}$ as with $\mathcal{A}$ in (11), i.e., $\mathcal{A}^{\prime} \triangleq \mathcal{H}^{\prime} \tilde{\mathbf{W}}$. For: 1$)$ $M \geq 2$; 2) $Q+D-P \geq L$; and 3 ) $\mathbf{h}$ has no null on any of the data carrier frequencies, it follows that $\mathbf{h}^{\prime}=\alpha \mathbf{h}$ where $\alpha$ is a complex scalar if $\operatorname{range}\left(\mathcal{A}^{\prime}\right)=\operatorname{range}(\mathcal{A})$.

Proof: See Appendix C.

Remarks on Theorems 1 and 2:

1) The presence of the VCs and/or CPs is necessary for the subspace method to work.

2) The condition $Q+D-L \geq P$ requires that the number of $\mathrm{VC}$ and/or $\mathrm{CP}$ be greater than the channel memory. It is much stronger than $M(Q+D-P) \geq L$ (this is necessary for $\mathcal{A}$ to be a tall matrix and consequent possible employment of the subspace method) to assure channel identifiability. This condition is satisfied in typical OFDM application scenarios for both CP-OFDM and non-CP OFDM.

3) Conditions for unique channel identification (up to an unknown complex scalar inherent in second-order blind methods) is guaranteed by the specific structure of $\mathcal{A}$ for $M \geq 2$. It is worth pointing out that (see the Appendix, proof of Theorem 2) such uniqueness is not possible for $M=1$, i.e., stacking the received signal over $M>1$ OFDM symbol durations is required.

4) Note that the cyclospectra [9], [10] or the finite alphabet property based methods [20] impose no restriction on the positions of channel spectral null, unlike Theorem 2 in our work. However, the probability that an exact spectral null is located on subcarriers is low; it is more likely that some sub carriers are subject to deep fades. Deep fading of subcarriers does not prevent identifiability but does pose issues regarding robustness of our algorithm — such algorithm robustness is verified by extensive computer experiments on a large number of random WSSUS channels.
Furthermore, we emphasize that no spectral null is only sufficient and not necessary condition for identifiability, as evidenced by simulation (not included in the paper) that channel with spectral null was uniquely identified via the proposed method.

5) As stated in Theorem 2, an amplitude/phase ambiguity exists in the channel estimate- this is inherent to all blind estimation approaches using second order statistics and cannot be resolved without further side information. Practical OFDM systems provide pilot tones for tracking the carrier frequency offset which can be exploited to resolve this ambiguity.

\section{B. Blind Channel Estimator}

Let

$$
\mathbf{U}_{n}(i)=\left[u_{i}(0), u_{i}(1), \ldots, u_{i}(M J-L-1)\right]^{T} .
$$

Exploiting the special structure of $\mathcal{H}$ yields

$$
\mathbf{U}_{n}(i)^{T} \mathcal{H}=\mathbf{h}^{T} \mathcal{U}_{i}
$$

where the $L+1 \times M J$ dimension matrix $\mathcal{U}_{i}$ is generated from the vector $\mathbf{U}_{n}(i)$

$$
\mathcal{U}_{i}=\text { Toeplitz }\left([\mathbf{U}_{n}^{T}(i) \underbrace{0 \cdots 0}_{L 0 \mathrm{~s}}]\right) .
$$

When only an estimate of the noise subspace $\hat{\mathbf{U}}_{n}$ is available in practice, (14) suggests the channel estimator

$$
\hat{\mathbf{h}}=\arg \min _{\|\mathbf{h}\|=1} \sum_{i=1}^{M(Q+D-P)-L}\left\|\hat{\mathbf{U}}_{n}(i)^{H} \mathcal{A}\right\|^{2} .
$$

But, from (16), we have

$$
\begin{aligned}
\left\|\mathbf{U}_{n}(i)^{H} \mathcal{A}\right\|^{2} & =\mathbf{U}_{n}(i)^{H} \mathcal{H} \tilde{\mathbf{W}} \tilde{\mathbf{W}}^{H} \mathcal{H}^{H} \mathbf{U}_{n}(i) \\
& =\mathbf{h}^{T} \underbrace{\left(\mathcal{U}_{i}\right)^{*} \tilde{\mathbf{W}}}_{\mathbf{G}_{i}} \underbrace{\tilde{\mathbf{W}}^{H} \mathcal{U}_{i}^{T}}_{\mathbf{G}_{i}^{H}} \mathbf{h}^{*} .
\end{aligned}
$$

Thus, by defining $\tilde{\mathbf{h}}=\mathbf{h}^{*}$, and

$$
\mathcal{G}=\left[\mathbf{G}_{1}, \ldots, \mathbf{G}_{M(Q+D-P)-L}\right]
$$

the channel information $\tilde{\mathbf{h}}$ is determined by

$$
\hat{\tilde{\mathbf{h}}}=\arg \min _{\|\tilde{\mathbf{h}}\|=1} \tilde{\mathbf{h}}^{H} \hat{\mathcal{G}} \hat{\mathcal{G}}^{H} \tilde{\mathbf{h}}
$$

where $\hat{\mathcal{G}}$ is the estimate of $\mathcal{G}$. It is well known that $\tilde{\mathbf{h}}$ (or equivalently $\mathbf{h}$ ) is the eigenvector corresponding to the smallest eigenvalue of the matrix $\hat{\mathcal{G}} \hat{\mathcal{G}}^{H}$.

\section{Simulation Results}

Monte Carlo simulations were conducted to assess the effectiveness of the proposed blind estimator with comparison to the other two subspace channel estimation methods for OFDM [9], [13]. To evaluate the estimation error, the normalized root mean square error (RMSE)

$$
\mathrm{RMSE}=\sqrt{\frac{1}{N_{m}(L+1)} \sum_{p=1}^{N_{m}} \frac{\left\|\hat{\mathbf{h}}_{p}-\mathbf{h}_{p}\right\|^{2}}{\left\|\mathbf{h}_{p}\right\|^{2}}}
$$


is used, where the subscript $p$ refers to the $p$ th simulation run and $N_{m}$ denotes the number of runs. Information sequence $s_{i}(k)$ 's are BPSK modulated. SNR is defined as SNR $\stackrel{\text { def }}{=} \log _{10}\left(E_{s} / E_{n}\right)$ for a fair comparison, where $E_{s}=P \sigma_{s}^{2}$ is the average OFDM symbol power and $E_{n}=J \sigma_{n}^{2}$ is the average noise power. The ambiguity in the channel estimate is resolved by assuming that the true channel vector $\mathbf{h}$ has unit norm and known phase of the first component. Thus, the estimate is normalized and the phase of $\left(h^{(1)}(0) / \hat{h}^{(1)}(0)\right)$ is used for phase ambiguity resolution and compensation prior to MSE computations.

The number of subcarriers used in simulations is $Q=15$, as used in [9] and [13]. The smoothing factor $M$ is set to 2 as in [13]. Multipath fading channels with order $L=3$ are generated according to Hoeher's method [21] by assuming an exponentially decaying power-delay profile $e^{-\left(\tau / \tau_{r m s}\right)}(\tau$ stands for path delay) with the rms delay ${ }^{2} \tau_{\text {rms }}=0.6 T$ and raised cosine pulse shaping with a roll-off factor 0.25 . Also, we set the maximum Doppler shift to 0 to get a time-invariant channel, which is plausible for burst packet transmission in wireless LAN applications. We tested all methods over 300 randomly generated channels, i.e., $N_{m}=300$. The average MSE results are shown below.

Example 1-Estimator's MSE Dependence on SNR or Data Record Length $N_{b}$ : As the proposed estimator is applicable to systems with or without $\mathrm{CP}$, it was tested on different system settings:

1) a system with no $\mathrm{CP}$ where $D=0$ and number of data carriers $P=11$;

2) system with insufficient $\mathrm{CP}$ where $D=2$ (less than channel order) and $P=13$;

3) system with sufficient $\mathrm{CP}$ where $D=4$ and $P=11$.

The results of those settings are then compared with the performance of the methods in [13] (marked "Cai") and [9] (marked "Heath"). Note that the estimators in [13] and [9] require the CP, whose length is set to 4 by convention (i.e., the number of $\mathrm{CP}$ equals as many as $25 \%$ of the number of total subcarriers).

Fig. 2 shows the estimator RMSE as a function of SNR for $N_{b}=300$, and Fig. 3 as a function of the number of data blocks $N_{b}$ for $\mathrm{SNR}=25 \mathrm{~dB}$. As expected, the estimator error of all methods decreases with increasing SNR and the data record length $N_{b}$. Also notable is that our approach and the methods in [13] perform better than that of [9] (marked "Heath"), reflecting the fast convergence property of the noise subspace estimator for small data record.

Additionally, for a fixed degree of freedom (that equals 4 in this example) through the combination of VCs and/or CPs, a performance gap exists between the non-CP system $(P=$ $11, D=0)$ and the CP-only system $(P=15, D=4)$ or the insufficient $\mathrm{CP}$ system $(P=13, D=2)$. That suggests that $\mathrm{CP}$ is more advantageous for the noise subspace-based estimator than $\mathrm{VC}$ is. However, the utilization of VCs provides the receiver with an extra source of redundancy other than $\mathrm{CP}$ and makes the proposed subspace method feasible for a system with insufficient $\mathrm{CP}(D=2$ in this example) without increasing the

\footnotetext{
${ }^{2} \mathrm{As} \tau_{\mathrm{rms}}=0.6 T$, a path with a delay larger than $3 T$ is negligible since it has an average power $20 \mathrm{~dB}$ lower than that of the path at the origin.
}

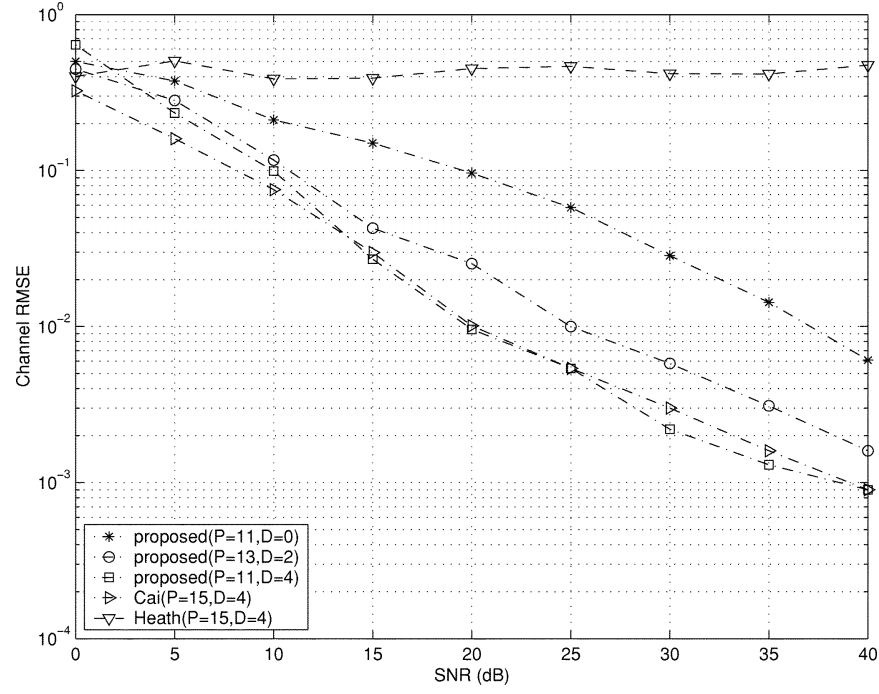

Fig. 2. RMSE versus SNR $\left(N_{b}=300\right)$.

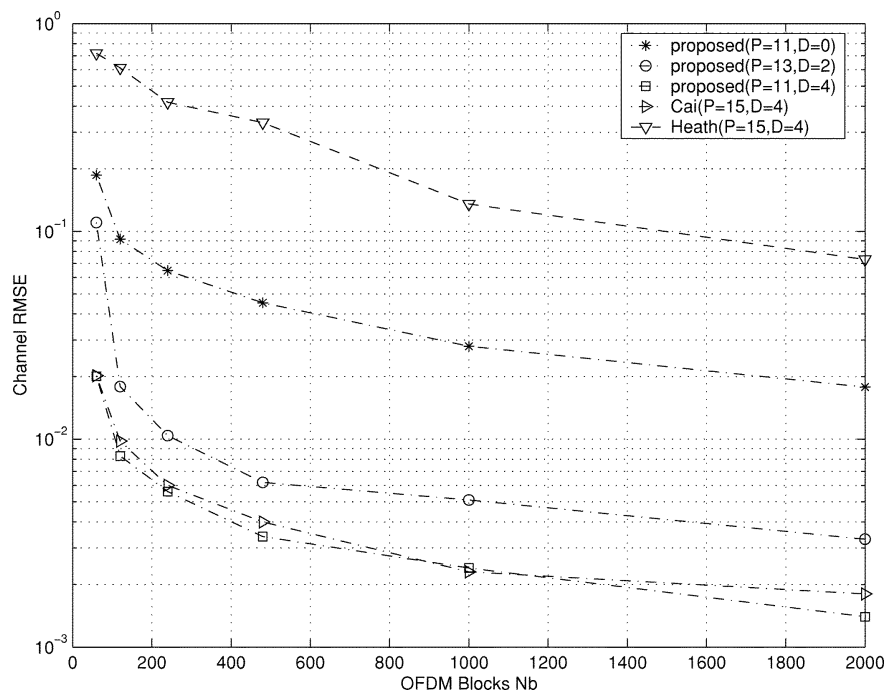

Fig. 3. RMSE versus number of data blocks $N_{b}(\mathrm{SNR}=25 \mathrm{~dB})$.

smoothing factor $M$. Note that $M$ must be increased in Cai's method, which means a larger observation dimension and a significant increase in computational complexity ${ }^{3}$ of the eigenstructure-based methods (such as ours and Cai's). Therefore, from the perspective of low computational complexity, it is desirable to exploit VC. Further, exploiting the additional redundancy of VCs for a sufficient CP system $(P=11, D=4)$ shows improved performance versus that of the CP-only system. Finally, it is worth reemphasizing that our proposed method is applicable to systems without $\mathrm{CP}$, potentially leading to higher throughput than [13].

From the next example, we will focus on our estimator that exploits the presence of $\mathrm{VC}$ and/or CP. Only results for purely non-CP system are shown since similar behavior is observed in systems with both VC and CP.

Example 2 - Robustness to Channel Order Overestimation: The proposed estimator is insensitive to the

${ }^{3}$ The computation complexity of eigenstructure-based methods is essentially $O\left[(M J-L)^{3}\right]$. 


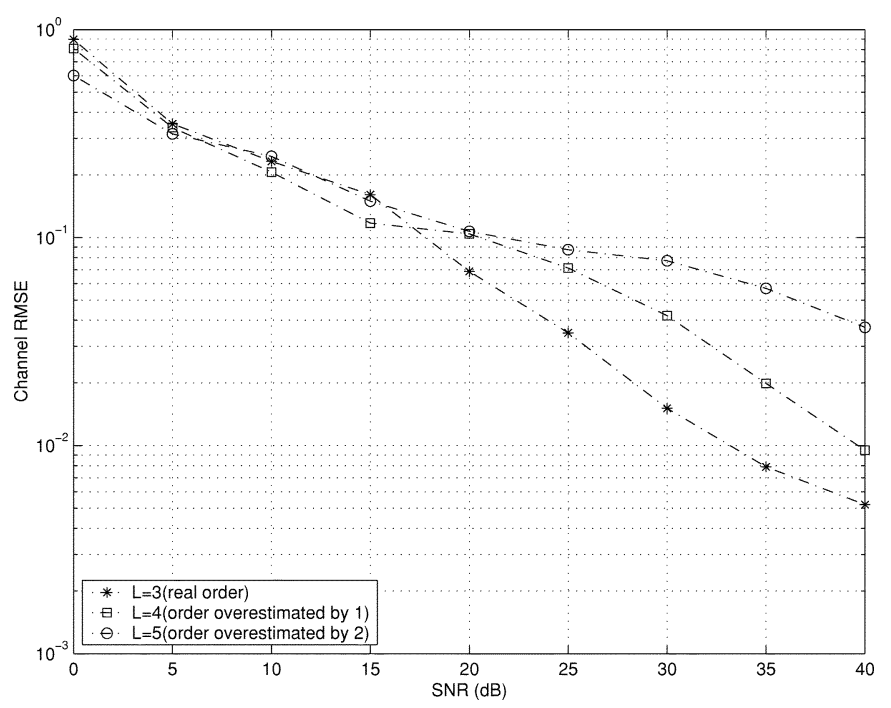

Fig. 4. RMSE versus SNR for different estimate of $L$ (non-CP system, $N_{b}=$ $300)$.

overestimation of $L$, as reflected by Fig. 4, where the real channel order $L=3$ is overestimated by 1 or 2 . The system assumes no $\mathrm{CP}$ insertion $(D=0)$ and the number of data carriers $P$ is set to 10 .

Example 3 - Impact of Number and Location of VCs: The dependence of the proposed estimator RMSE on the number and the location of the available VCs is highlighted in Fig. 5, using $\mathrm{SNR}=25 \mathrm{~dB}$ and $N_{b}=300$. The system assumes no CP also, and the number of VCs is varied from 3 to 7 (correspondingly, the number of the data carriers $P$ goes from 12 to 8 ). Results corresponding to three different locations - on the low-frequency side of the spectrum only $\left(p_{0}=Q-P\right.$, marked as "left"), on the high-frequency side of the spectrum only $\left(p_{0}=0\right.$, marked as "right"), and on both sides of the spectrum $\left(p_{0}=\right.$ $\lfloor(Q-P) / 2\rfloor$, marked as "center")—are plotted together. From (14), an increase in the number of VCs increases the ratio of the noise subspace dimension to the observation space dimension $[(M(Q-P)-L) /(M Q-L)$ in this case $]$, when the number of subcarriers and the channel order are fixed. This increase is advantageous for improving estimation accuracy as it imposes more constraints on the channel order for estimation as seen in Fig. 5. Note that no transmit power normalization is employed (i.e., a transmitted OFDM symbol has constant energy irrespective of the number of data carriers). In case of power normalization, the increase in number of VCs means more power is allocated to each of the fewer data carriers, thus leading to further performance improvement. While it is observed that the location of VCs does affect the performance, the impact is small.

\section{CONCLUSION}

In this paper, we presented a subspace-based blind channel estimator for OFDM systems with or without CP by exploiting the presence of the VCs. A sufficient condition for identifiability was established as well. The algorithm is attractive due to its potential for enhancing channel utilization as it can be applied to OFDM systems with insufficient $\mathrm{CP}$ or even without CP. Comparison of the proposed method with other reported subspace

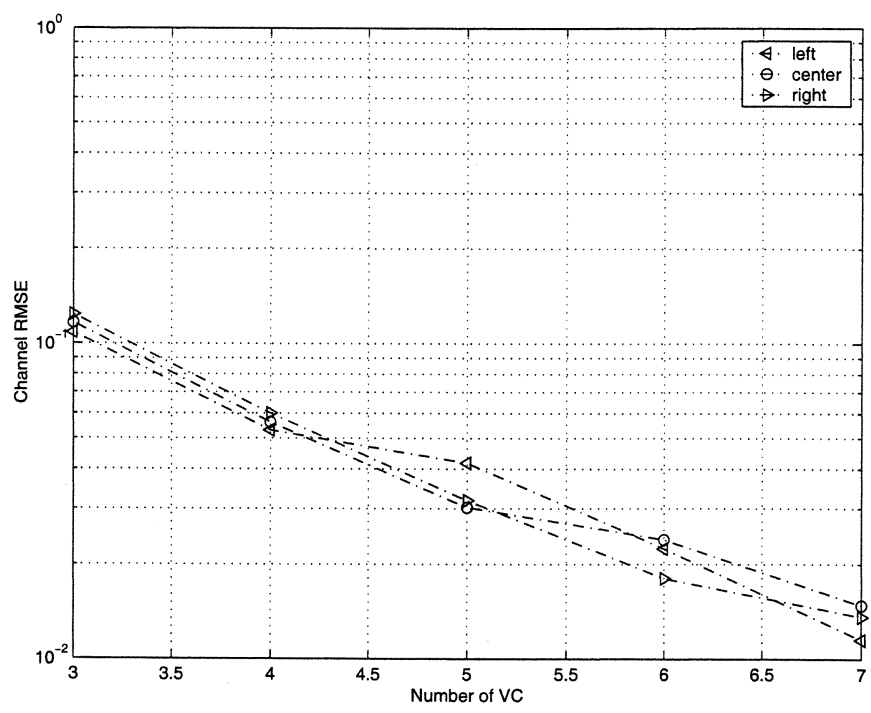

Fig. 5. RMSE versus the number of VC.

channel estimation methods by computer simulation illustrates effectiveness of the proposed method with regard to both estimation accuracy and speed of convergence.

\section{APPENDIX}

\section{PROOF OF IDENTIFIABILITY CONDITIONS}

\section{A. Some Important Relations}

First, some important preliminary results are stated prior to the proofs of Theorems 1 and 2 .

Let the frequency response of the channel coefficients ${ }^{4}$ $[h(0), \ldots, h(L)]^{T}$ on the active data carriers be the $P \times 1$ vector

$$
\begin{aligned}
& \mathbf{H}=\left[H\left(p_{0}\right), \ldots, H\left(p_{0}+P-1\right)\right]^{T}
\end{aligned}
$$

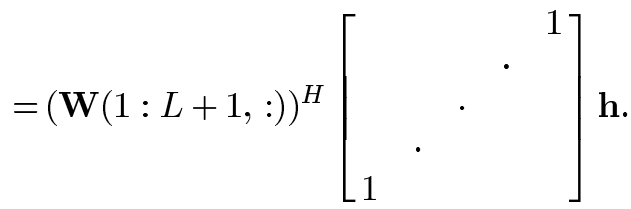

A "rotated" version of the frequency response denoted by $\mathbf{g}$ is given by

$$
\begin{aligned}
& \mathbf{g}=[g(0), \ldots, g(P-1)]^{T} \\
& =\left[\begin{array}{ccc}
w_{Q}^{-p_{0} L} & & \\
& \ddots & \\
& & w_{Q}^{-\left(p_{0}+P-1\right) L}
\end{array}\right] \mathbf{H} \\
& =\left[\begin{array}{c}
h(L)+h(L-1) w_{Q}^{-p_{0}}+\cdots+h(0) w_{Q}^{-p_{0} L} \\
\vdots \\
h(L)+h(L-1) w_{Q}^{-\left(p_{0}+P-1\right)}+\cdots+h(0) w_{Q}^{-\left(p_{0}+P-1\right) L}
\end{array}\right]
\end{aligned}
$$

The structure of the matrices $\mathcal{H}$ (Toeplitz) and $\overline{\mathbf{W}}$ allows the first $J-L$ elements in the $i$ th $(i=1, \ldots, P)$ column of $\mathcal{A}$ to

${ }^{4}$ Note that the channel vector $\mathbf{h}$ is defined throughout as $[h(L), \ldots, h(0)]^{T}$. 
be given as shown in (26), at the bottom of the page. Hence, it is easy to see that the following relation holds that connects the channel "rotated" frequency response vector $\mathrm{g}$ to the submatrix $\mathcal{A}(1: J-L, 1: P)$ :

$$
\begin{aligned}
\mathcal{A} & (1: J-L, 1: P) \\
& =\mathcal{H}(1: J-L, 1: J) \overline{\mathbf{W}} \\
& =\sqrt{Q} \overline{\mathbf{W}}(1: J-L,:) \underbrace{\left[\begin{array}{rrr}
g(0) & \\
& \ddots & \\
& & g(P-1)
\end{array}\right]}_{\mathbf{G}} \\
& =\sqrt{Q} \overline{\mathbf{W}}(1: J-L,:) \mathbf{G} .
\end{aligned}
$$

In order to further reveal the relation between $\mathbf{h}$ and column rank of filtering matrix $\mathcal{A}$, we next transform $\mathcal{A}$ to some other forms which are useful in later derivations.

Define two $L \times L$ triangle matrices

$$
\begin{aligned}
H_{u} & =\left[\begin{array}{ccc}
h(L) & \ldots & h(1) \\
& \ddots & \vdots \\
& & h(L)
\end{array}\right] \\
H_{d} & =\left[\begin{array}{ccc}
h(0) & & \\
\vdots & \ddots & \\
h(L-1) & \ldots & h(0)
\end{array}\right]
\end{aligned}
$$

and two $L \times P$ rectangular matrices

$$
\begin{aligned}
\tilde{H}_{u} & =H_{u} \overline{\mathbf{W}}(J-L+1: J,:) \\
\tilde{H}_{d} & =H_{d} \overline{\mathbf{W}}(1: L,:) .
\end{aligned}
$$

By exercising the structure exhibited by (27) and the expression (30), submatrix $\mathcal{A}(1: J, 1: P)$ can be written as

$$
\begin{aligned}
& \mathcal{A}(1: J, 1: P) \\
& =\mathcal{H}(1: J, 1: J) \overline{\mathbf{W}}
\end{aligned}
$$

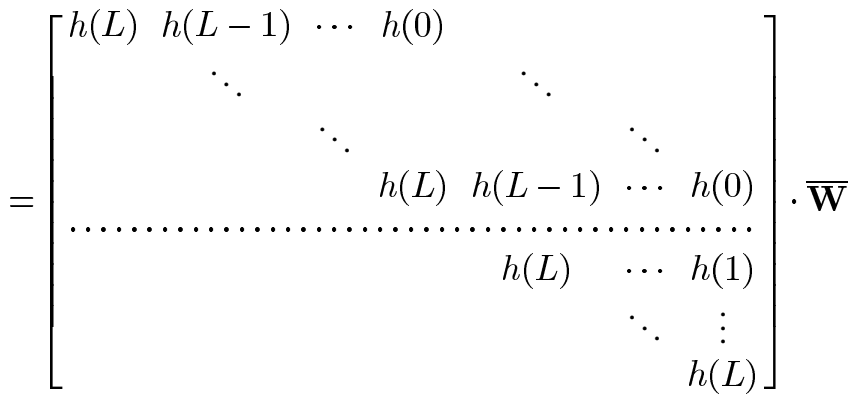

$$
\begin{aligned}
& =\left[\begin{array}{c}
\sqrt{Q} \overline{\mathbf{W}}(1: J-L,:) \mathbf{G} \\
\cdots \ldots \ldots \ldots \ldots \ldots \ldots \\
\tilde{H}_{u}
\end{array}\right] \text {. }
\end{aligned}
$$

Thus, we can partition the filtering matrix $\mathcal{A}$ as shown in (33), at the bottom of the next page.

In the sequel, we give proofs for the two theorems, where the structure exhibited in (24) and (33) will be used repeatedly.

\section{B. Proof of Theorem 1}

Theorem 1: For $(Q+D-L) \geq P, \mathcal{A}$ has full column rank (i.e., $\operatorname{rank}(\mathcal{A})=M P$ ), if and only if channel frequency response has no nulls at any of the data subcarrier frequencies.

Proof:

Sufficiency: For $Q+D-L \geq P$, we can extract the rows $(m-1) J+1$ to $(m-1) J+P$ (recall $J=Q+D)$ for $m=1, \ldots, M$ from $\mathcal{A}$ [see (33)] to yield an $M P \times M P$ submatrix $\tilde{\mathcal{A}}$

$$
\begin{aligned}
& \tilde{\mathcal{A}}=\sqrt{Q} \\
& .\left[\begin{array}{llll}
\overline{\mathbf{W}}(1: P,:) \mathbf{G} & & \\
& \overline{\mathbf{W}}(1: P,:) \mathbf{G} & & \\
& & \ddots & \\
& & & \overline{\mathbf{W}}(1: P,:) \mathbf{G}
\end{array}\right] .
\end{aligned}
$$

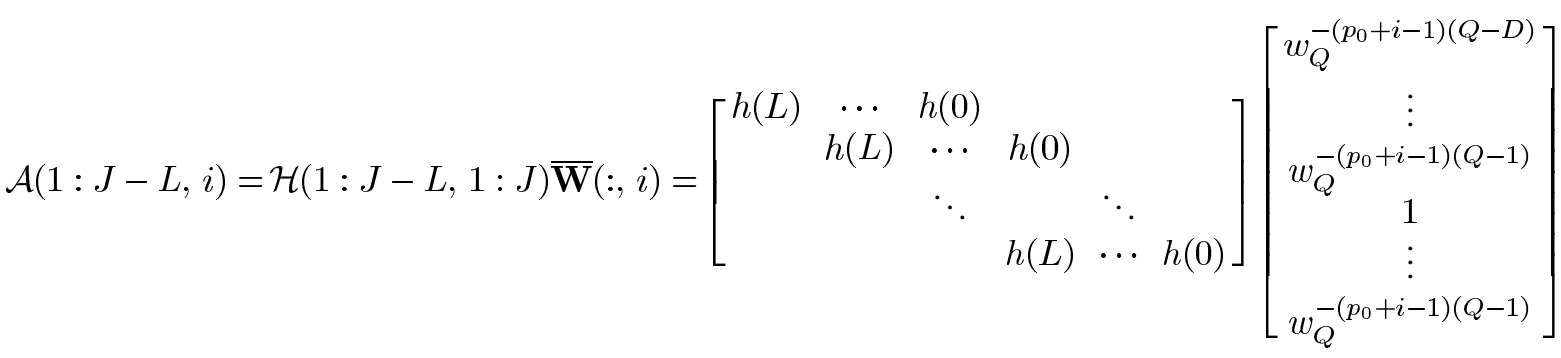

$$
\begin{aligned}
& =\left[\begin{array}{c}
h(L) w_{Q}^{-\left(p_{0}+i-1\right)(Q-D)}+\cdots+h(0) w_{Q}^{-\left(p_{0}+i-1\right)(Q-D+L)} \\
\vdots \\
h(L) w_{Q}^{-\left(p_{0}+i-1\right)(Q-L-1)}+\cdots+h(0) w_{Q}^{-\left(p_{0}+i-1\right)(Q-1)}
\end{array}\right] \\
& =\left[\begin{array}{c}
w_{Q}^{-\left(p_{0}+i-1\right)(Q-D)}\left(h(L)+\cdots+h(0) w_{Q}^{-\left(p_{0}+i-1\right) L}\right) \\
\vdots \\
w_{Q}^{-\left(p_{0}+i-1\right)(Q-L-1)}\left(h(L)+\cdots+h(0) w_{Q}^{-\left(p_{0}+i-1\right) L}\right)
\end{array}\right]=\overline{\mathbf{W}}(1: J-L, i) \cdot g(i)
\end{aligned}
$$


Note that the structure in $\overline{\mathbf{W}}(1: P,:)$ allows

$$
\begin{aligned}
\overline{\mathbf{W}}(1: P,:)= & \frac{1}{\sqrt{Q}}\left[\begin{array}{ccc}
1 & \cdots & 1 \\
w_{Q}^{-p_{0}} & \cdots & w_{Q}^{-\left(p_{0}+P-1\right)} \\
\vdots & & \vdots \\
w_{Q}^{-p_{0}(P-1)} & \cdots & w_{Q}^{-\left(p_{0}+P-1\right)(P-1)}
\end{array}\right] \\
& \cdot\left[\begin{array}{ccc}
w_{Q}^{-p_{0}(Q-D)} & & \\
& \ddots & w_{Q}^{-\left(p_{0}+P-1\right)(Q-D)}
\end{array}\right] .
\end{aligned}
$$

The first matrix on the right in (35) is a Vandermonde matrix with unique elements, thus $\overline{\mathbf{W}}(1: P,:)$ has full column rank $(P)$. If the channel has no nulls at any of the data subcarrier frequencies, $\mathbf{G}$ is nonsingular by (24). Therefore, the matrix $\overline{\mathbf{W}}(1: P,:) \mathbf{G}$ and consequently $\tilde{\mathcal{A}}$ are nonsingular or, equivalently, $\operatorname{rank}(\tilde{\mathcal{A}})=M P$.

Since the submatrix $\tilde{\mathcal{A}}$ results from $\mathcal{A}$ by row deletion, the rank inequality condition [22] leads to $M P=\operatorname{rank}(\tilde{\mathcal{A}}) \leq$ $\operatorname{rank}(\mathcal{A}) \leq M P$, i.e., the filtering matrix $\mathcal{A}$ has full column rank.

Necessity: We now show that if the channel has spectral null at a data subcarrier location, then $\mathcal{A}$ cannot be full column rank. We remark that showing necessity does not readily follow from the structure of $\mathcal{A}$ in (33), and an alternate form (as below) is needed.

Let $\mathbf{T}$ be an $M P \times M P$ lower triangle matrix that has the following structure: $1 \mathrm{~s}$ on the main diagonal and $1_{(M-m)} \otimes$ $\left[w_{Q}^{-m D p_{0}}, \ldots, w_{Q}^{-m D\left(p_{0}+P-1\right)}\right]$ as the $m P$ th subdiagonals for $m=1, \ldots M-1$; all other elements are zero. It is obvious that $\mathbf{T}$ is nonsingular. Next we transform $\mathcal{A}$ to a new $M J-L \times M P$ matrix

$$
\breve{\mathcal{A}}=\mathcal{A} \cdot \mathbf{T}
$$

which has the same column rank as $\mathcal{A}$. The first $P$ columns of $\breve{\mathcal{A}}$ are shown in (37), at the bottom of the page. $\breve{\mathcal{A}}(: .1, P)$ in (37) directly reveals that, if any element of the diagonal matrix $\mathbf{G}$ is zero (equivalently, a null data subcarrier exists), then it is column rank deficient. Thus, necessity of the condition that the channel $\mathbf{h}$ has no spectral null at any of the data subcarriers for filtering matrix $\mathcal{A}$ to be full column rank is established.

\section{Proof of Theorem 2}

Theorem 2: Let $\mathbf{h}^{\prime}, \mathbf{h}$ be distinct $L+1$ vectors; the matrix $\mathcal{A}^{\prime}$ is constructed using $\mathbf{h}^{\prime}$ as with $\mathcal{A}$ in (11), i.e., $\mathcal{A}^{\prime} \triangleq \mathcal{H}^{\prime} \tilde{\mathbf{W}}$.

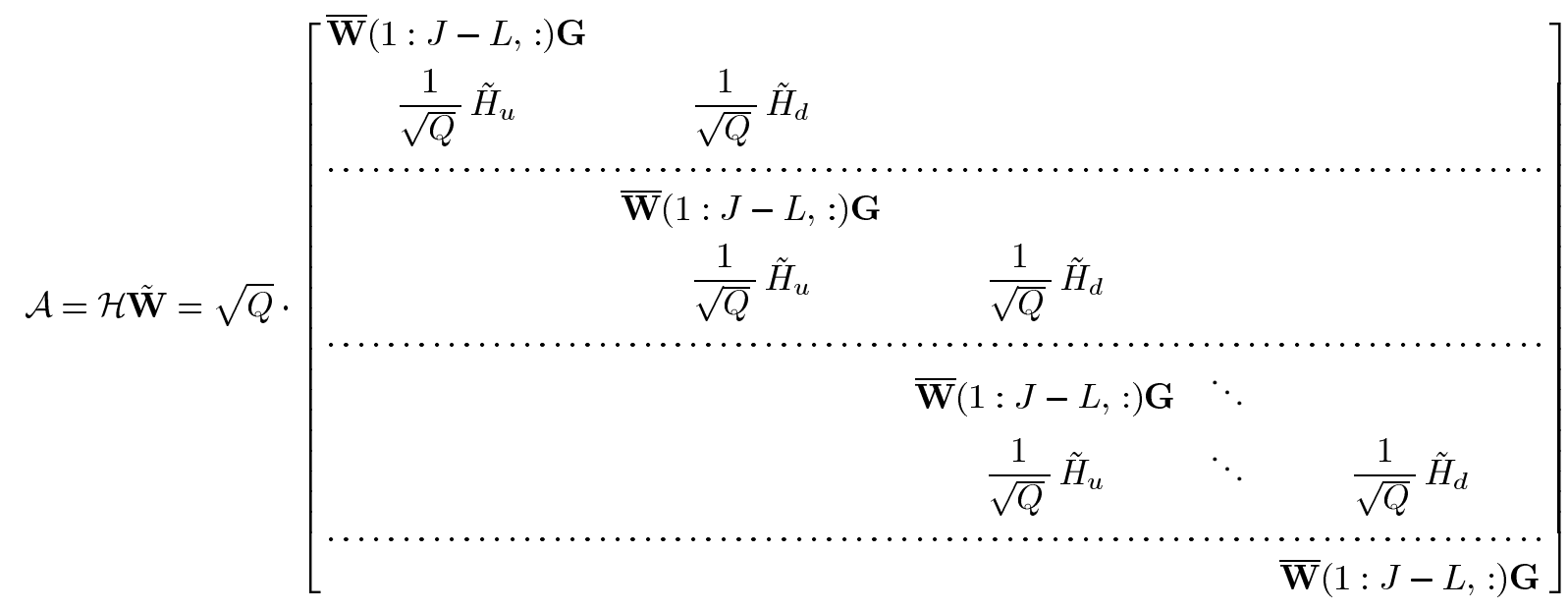

$$
\breve{\mathcal{A}}(:, 1: P)=\mathcal{A} \mathbf{T}(:, 1: P)=\sqrt{Q} \cdot\left[\begin{array}{c}
\overline{\mathbf{W}}(1: J-L,:) \mathbf{G} \\
\overline{\mathbf{W}}(J-L+1: J,:) \mathbf{G} \\
\overline{\mathbf{W}}(1: J-L,:) \mathbf{G} \operatorname{diag}\left(\left[w_{Q}^{-D p_{0}}, \ldots, w_{Q}^{-D\left(p_{0}+P-1\right)}\right]\right) \\
\overline{\mathbf{W}}(J-L+1: J,:) \mathbf{G} \operatorname{diag}\left(\left[w_{Q}^{-D p_{0}}, \ldots, w_{Q}^{-D\left(p_{0}+P-1\right)}\right]\right) \\
\overline{\mathbf{W}}(1: J-L,:) \mathbf{G} \operatorname{diag}\left(\left[w_{Q}^{-2 D p_{0}}, \ldots, w_{Q}^{-2 D\left(p_{0}+P-1\right)}\right]\right) \\
\overline{\mathbf{W}}(J-L+1: J,:) \mathbf{G} \operatorname{diag}\left(\left[w_{Q}^{-2 D p_{0}}, \ldots, w_{Q}^{-2 D\left(p_{0}+P-1\right)}\right]\right) \\
\vdots \\
\overline{\mathbf{W}}(1: J-L,:) \mathbf{G} \operatorname{diag}\left(\left[w_{Q}^{-(M-1) D p_{0}}, \ldots, w_{Q}^{-(M-1) D\left(p_{0}+P-1\right)}\right]\right)
\end{array}\right]
$$


For: 1) $M \geq 2$; 2) $Q+D-P \geq L$; and 3) $\mathbf{h}$ has no null at any of the data carrier frequencies, if $\operatorname{range}\left(\mathcal{A}^{\prime}\right)=\operatorname{range}(\mathcal{A})$, then $\mathbf{h}^{\prime}=\alpha \mathbf{h}$ where $\alpha$ is a complex scalar.

Comments: It is worth noting the importance of the three conditions 1)-3). According to Theorem 1, assumptions 2) and 3 ) ensure that $\mathcal{A}$ will be full column rank. The new requirement that $M \geq 2$ arises from the fact that for $M=1, \mathbf{h}$ cannot be uniquely determined. This is clear by studying the structure of $\mathcal{A}$ for the case $M=1$, which is now a $J-L \times P$ matrix

$$
\mathcal{A}=\mathcal{H}(1: J-L, 1: J) \overline{\mathbf{W}}=\sqrt{Q} \overline{\mathbf{W}}(1: J-L,:) \mathbf{G}
$$

where the second equation is essentially (27). The above implies that the signal subspace is completely determined by the columns of $\overline{\mathbf{W}}(1: J-L,:)$, which is known. Therefore, the noise subspace method (14) in this case is not able to give a unique solution to $\mathbf{h}$. Hence, in the sequel we assume that $M \geq 2$.

Proof: Define an $M J-L \times M J-L$ nonsingular matrix $\mathbf{F}$ whose main diagonal elements are $1 \mathrm{~s}$ and

$\mathbf{F}(J+2: J+P, J+1)$

$$
=-\left[w_{Q}^{-p_{0}}, w_{Q}^{-2 p_{0}}, \ldots, w_{Q}^{-(P-1) p_{0}}\right]^{T} .
$$

All other elements of $\mathbf{F}$ are zeros.

Left-multiplying $\mathbf{F}$ by $\mathcal{A}$ and $\mathcal{A}^{\prime}$ yields $M J-L \times M P$ matrices $\mathcal{B}=\mathbf{F} \mathcal{A}$ and $\mathcal{B}^{\prime}=\mathbf{F} \mathcal{A}^{\prime}$. For $\mathbf{F}$ being full rank, the knowledge of the column space of $\mathcal{B}$ characterizes $\mathcal{A}$ up to a scalar constant and thus [15]

$$
\operatorname{range}\left(\mathcal{A}^{\prime}\right)=\operatorname{range}(\mathcal{A}) \Leftrightarrow \operatorname{range}\left(\mathcal{B}^{\prime}\right)=\operatorname{range}(\mathcal{B}) \text {. }
$$

By exercising the structure of $\mathbf{F}$ and $\mathcal{A}$ [namely, (39) and (33) again], $\mathcal{B}$ can be partitioned as shown in (41), at the bottom of the page, where $\mathbf{B}_{1}=\sqrt{Q} \overline{\mathbf{W}}(1: J-L,:) \mathbf{G}$ is a $J-L \times P$ matrix, $\mathbf{B}_{4}$ is a $P-1 \times P-1$ matrix as

$$
\begin{aligned}
\mathbf{B}_{4}=\sqrt{Q} & \cdot \mathbf{F}(J+2: J+P, J+1: J+P) \\
\cdot & \overline{\mathbf{W}}(1: P, 2: P) \cdot\left[\begin{array}{lll}
g(1) & & \\
& \ddots & \\
& & g(P-1)
\end{array}\right]
\end{aligned}
$$

the $L+1 \times 1$ vector $\mathbf{b}$ (which is part of the $P+1$ th column of $\mathcal{B}$ ) is the product of a right lower triangle matrix and the channel vector $\mathbf{h}$, as shown in (43) at the bottom of the page, and $\mathbf{B}_{2}, \mathbf{B}_{3}, \mathbf{B}_{5}, \mathbf{B}_{6}, \mathbf{B}_{7}, \mathbf{B}_{8}$ are submatrices with conformable dimensions.

From the proof of Theorem 1, $\mathbf{B}_{1}((J-L) \times P)$ is full column rank. Next we will show $\mathbf{B}_{4}$ that is full rank as well. Defining a $P-1 \times 1$ vector $\mathbf{e}$ where

$$
\mathbf{e}=\left[\begin{array}{lll}
g(1) & & \\
& \ddots & \\
& & g(P-1)
\end{array}\right] \cdot(\overline{\mathbf{W}}(1,2: P))^{T}
$$

the $P \times P$ submatrix $\mathcal{B}(J+1: J+P, P+1: 2 P)$ can be written as

$$
\begin{aligned}
& \mathcal{B}(J+1: J+P, P+1: 2 P) \\
& =\left[\begin{array}{cccc}
1 & & & \\
-w_{Q}^{-p_{0}} & 1 & & \\
\vdots & & \ddots & \\
-w_{Q}^{-p_{0}(P-1)} & & & 1
\end{array}\right] \cdot \sqrt{Q} \overline{\mathbf{W}}(1: P,:) \cdot \mathbf{G} \\
& =\left[\begin{array}{cc}
w_{Q}^{-p_{0}(Q-D)} g(0) & \mathbf{e}^{T} \\
0_{(P-1) \times 1} & \mathbf{B}_{4}
\end{array}\right] .
\end{aligned}
$$

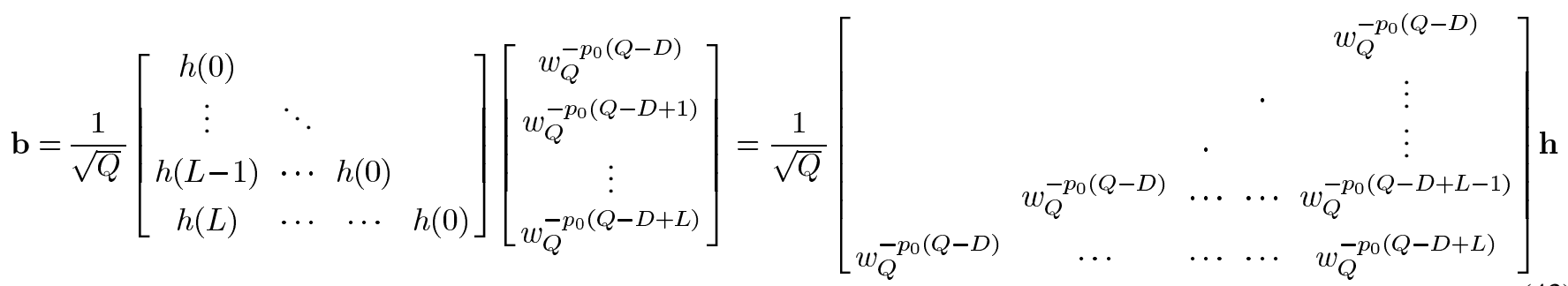


It can be seen that, from (45), the submatrix is full rank (once again note that $\overline{\mathbf{W}}(1: P,:)$ is full rank). Furthermore, (46) yields $\operatorname{det}\left(\mathbf{B}_{4}\right) \cdot w_{Q}^{-p_{0}(Q-D)} g(0)=\operatorname{det}(\mathcal{B}(J+1: J+P, P+1$ : $2 P)) \neq 0$, where $\operatorname{det}(\cdot)$ gives the determinant. Hence, $\mathbf{B}_{4}$ is full rank.

Since $\operatorname{range}\left(\mathcal{B}^{\prime}\right)=\operatorname{range}(\mathcal{B})$, the vector $\left[\mathbf{0}_{J-L}^{T},\left(\mathbf{b}^{\prime}\right)^{T}\right.$, $\left.\mathbf{0}_{P-1}^{T},\left(\mathbf{B}_{5}^{\prime}\right)^{T}, \mathbf{0}_{(M-2) J-L}^{T}\right]^{T}$, which is the $P+1$ th column of $\mathcal{B}^{\prime}$, belongs to $\operatorname{range}(\mathcal{B})$ also, namely

$$
\left[\begin{array}{c}
\mathbf{0}_{J-L} \\
\mathbf{b}^{\prime} \\
\mathbf{0}_{P-1} \\
\mathbf{B}_{5}^{\prime} \\
\mathbf{0}_{(M-2) J-L}
\end{array}\right]=\mathcal{B} \cdot\left[\begin{array}{c}
\mathbf{q}_{1} \\
\alpha \\
\mathbf{q}_{2} \\
\mathbf{q}_{3}
\end{array}\right]
$$

where $\mathbf{q}_{1}, \mathbf{q}_{2}, \mathbf{q}_{3}$ are $P \times 1, P-1 \times 1$, and $(M-2) P \times 1$ vectors while $\alpha$ is a scalar. The above implies

$$
\begin{aligned}
\mathbf{0}_{J-L} & =\mathbf{B}_{1} \mathbf{q}_{1} \\
\mathbf{b}^{\prime} & =\mathbf{b} \alpha+\mathbf{B}_{2} \mathbf{q}_{1}+\mathbf{B}_{3} \mathbf{q}_{2} \\
\mathbf{0}_{P-1} & =\mathbf{B}_{4} \mathbf{q}_{2} .
\end{aligned}
$$

The full column rank property of $\mathbf{B}_{1}$ and $\mathbf{B}_{4}$ yields $\mathbf{q}_{1}=0$ and $\mathbf{q}_{2}=0$. Therefore, $\mathbf{b}^{\prime}=\mathbf{b} \alpha$ or, equivalently, by the structure of (43), $\mathbf{h}^{\prime}=\alpha \mathbf{h}$. This concludes the proof of Theorem 2 .

\section{REFERENCES}

[1] J. A. C. Bingham, "Multicarrier modulations for data transmission: An idea whose time has come," IEEE Commun. Mag., vol. 28, pp. 5-14, May 1990.

[2] W. Y. Zou and Y. Wu, "COFDM: An overview," IEEE Trans. Broadcasting, vol. 41, pp. 1-8, Mar. 1995.

[3] Wireless LAN Medium Access Control (MAC) and Physical Layer (PHY) specifications: High speed physical layer in the 5 GHZ band, IEEE $80211 \mathrm{a}, 1999$.

[4] H. Sari, G. Karam, and I. Jeanclaude, "Transmission techniques for digital terrestrial TV broadcasting," IEEE Commun. Mag., vol. 33, pp. 100-109, Feb. 1995.

[5] T. Pollet, M. Peeters, M. Moonen, and L. Vandendorpe, "Equalization for DMT-based broadband modems," IEEE Commun. Mag., vol. 38, pp. 106-113, May 2000.

[6] D. Ikonomou and L. Vandendorpe, "A fractionally spaced decision feedback joint detection proposal for multi carrier CDMA," Eur. Trans. Telecommun., vol. 10, no. 4, pp. 407-416, July-Aug. 1999.

[7] O. Edfors, M. Sandell, J. van de Beek, S. Kate, and P. O. Borjesson, "OFDM channel estimation by singular value decomposition," IEEE Trans. Commun., vol. 46, pp. 931-939, July 1998.

[8] Y. Li, L. J. Cimini, Jr. , and N. R. Sollenberger, "Robust channel estimation for OFDM systems with rapid dispersive fading channels," IEEE Trans. Commun., vol. 46, pp. 902-915, July 1998.

[9] R. W. Heath and G. B. Giannakis, "Exploiting Input Cyclostationarity for blind channel identification in OFDM systems," IEEE Trans. Signal Processing, vol. 47, pp. 848-856, Mar. 1999.

[10] B. Muquet and M. de Courville, "Blind and semi-blind channel identification methods using second order statistics for OFDM systems," in Proc. ICASSP 1999, vol. 5, 1999, pp. 2745-2748.
[11] X. Zhuang, Z. Ding, and A. L. Swindlehurst, "A statistical subspace method for blind channel identification in OFDM communications," in Proc. ICASSP 2000, vol. 5, 2000, pp. 2493-2496.

[12] B. Muquet, M. de Courville, P. Duhamel, and V. Buenac, "A subspace based blind and semi-blind channel identification method for OFDM systems," in Proc. IEEE-SP Workshop on Signal Proc. Advances in Wireless Comm. 1999, Annapolis, MD, May 1999, pp. 170-173.

[13] X. Cai and A. N. Akansu, "A subspace method for blind channel identification in OFDM systems," in Proc. ICC2000, 2000, pp. 929-933.

[14] X. Wang and K. J. Ray Liu, "Adaptive channel estimation using cyclic prefix in multicarrier modulation system," IEEE Commun. Lett., vol. 3, pp. 291-293, Oct. 1999.

[15] E. Moulines, P. Duhamel, J. Cardoso, and S. Mayrargue, "Subspace methods for the blind identification of multichannel FIR filters," IEEE Trans. Signal Processing, vol. 43, pp. 516-525, Feb. 1995.

[16] M. de Courville, P. Madec, and J. Palicot, "Blind equalization of OFDM system based on the minimization of a quadratic criterion," in Proc. ICC '96, 1996, pp. 1318-1322.

[17] U. Tureli and H. Liu, "A high-efficiency carrier estimator for OFDM communications," IEEE Commun. Lett., vol. 2, pp. 104-106, Apr. 1998.

[18] Y. Song, S. Roy, and L. A. Akers, "Joint blind estimation of channel and data symbols in OFDM," in Proc. VTC Spring 2000, 2000, pp. 46-50.

[19] C. Li and S. Roy, "A subspace blind channel estimation method for OFDM systems without cyclic prefix," in Proc. VTC Fall 2001, Oct. 2001, pp. 2148-2152.

[20] S. Zhou and G. B. Giannakis, "Finite-alphabet based channel estimation for OFDM and related multicarrier systems," IEEE Trans. Commun., vol. 49, pp. 1402-1414, Aug. 2001.

[21] P. Hoeher, "A statistical discrete-time model for the WSSUS multipath channel,” IEEE Trans. Veh. Technol., vol. 41, pp. 461-468, Nov. 1992.

[22] R. A. Horn and C. R. Johnson, Matrix Analysis. Cambridge, U.K.: Cambridge Univ. Press, 1985, p. 13.

Chengyang Li (S'01-M'02) received the B.S. and M.S. degrees from the University of Electronic Science and Technology of China, Chengdu, in 1992 and 1995, respectively, both in electrical engineering. He is currently working toward the Ph.D. degree at the University of Washington, Seattle.

Since 1999, he has been a Research Assistant at the FUndamentals of Networking LABoratory (FUNLAB), University of Washington. His research interests include OFDM/multicarrier CDMA systems, multiuser communications, and wireless LAN.

Sumit Roy (S'84-M'88-SM'00) received the B.Tech. degree from the Indian Institute of Technology, Kanpur, in 1983 and the M.S. and Ph.D. degrees from the University of California, Santa Barbara, in 1985 and 1988, respectively, all in electrical engineering, as well as the M.A. degree in statistics and applied probability in 1988 .

His previous academic appointments were at the Moore School of Electrical Engineering, University of Pennsylvania, Philadelphia, and at the University of Texas, San Antonio. Presently, he is a Professor of Electrical Engineering, University of Washington, Seattle, where his research interests include analysis/design of communication systems/networks, with a topical emphasis on next generation mobile/wireless networks. He is currently on academic leave at Intel Wireless Technology Lab working on ultrawideband radios for high-speed wireless access.

Prof. Roy is a member of the IEEE Communications Society and is on several technical committees and conference program committees. He presently serves as an Editor for the IEEE TRANSACTIONS ON WIRELESS COMMUNICATIONS. 